ISSN 1991-8631

Original Paper

http://indexmedicus.afro.who.int

\title{
Influence des perturbations anthropiques sur la biodiversité des bois sacrés du Centre Bénin
}

\author{
Romaric Iralè EHINNOU KOUTCHIKA *, Pierre Onodjè AGBANI et Brice SINSIN \\ Laboratoire d'Ecologie Appliquée-Faculté des Sciences Agronomiques-Université d'Abomey-Calavi, \\ 01 BP 526 Cotonou, Bénin ; Fax:00229 21303084 ; Tél : (+229) 900356 18/(+229) 96080709 \\ *Auteur correspondant, E-mail : akofoudi2004@yahoo.fr
}

\section{RESUME}

Le Centre Bénin couvre les Communes de Glazoué, de Savè et de Ouèssè. L'étude a ciblé 61 bois sacrés autour desquels des entretiens ont été réalisés avec les populations riveraines. A cet effet, 244 personnes de façon raisonnée ont été interrogées. Ces entretiens ont permis de savoir que les valeurs traditionnelles, culturelles, alimentaires et thérapeutiques ont un intérêt pour les communautés. Les différents entretiens avec les populations locales ont permis de comprendre qu'elles accordent du prix aux bois sacrés. Ces lieux sacrés constituent l'habitat des espèces animales et végétales rares et menacées de disparition. Les interdits liés à ces bois sacrés permettent de conserver leur biodiversité. Les relevés phytosociologiques réalisés sur des placettes de $30 \mathrm{~m}$ x $30 \mathrm{~m}$ ont permis de savoir que $47 \%$ des bois sacrés sont sous pression pastorale. Dans ces bois sacrés, 7 espèces (Afzelia africana , Borassus aethiopum,Khaya senegalensis, Milicia excelsa, Ptereocarpus erinaceus, Vitellaria paradoxa et Zanthoxylum zanthozyloides) sont sur la liste rouge de l'UICN. Le statut des espèces au Bénin montre que 4 espèces sont en danger et 3 espèces sont vulnérables. Dans ces bois sacrés, 12 espèces animales et 25 espèces floristiques sont citées dans le traitement de certaines maladies.

(C) 2013 International Formulae Group. All rights reserved.

Mots clés: Communautés, bois sacrés, valeurs culturelles, maladies, Centre Bénin.

\section{INTRODUCTION}

Les bois sacrés d'Afrique, d'Amérique latine et d'Asie intéressent désormais les scientifiques et les organismes de protection de la nature. Certains pensent que leur sauvegarde peut représenter une solution pour la conservation de la diversité biologique. $\mathrm{Au}$ Bénin et au Togo, les reliques de végétations forestières anciennes et de leur diversité biologique, ont fait l'objet de plusieurs études (Kokou et Caballé, 2000 ; Kokou et Sokpon, 2006 ; Kokou et Kokutse, 2010). Ces bois sacrés sont encore considérés comme les reliques d'une végétation forestière primaire ou climacique restée inchangée grâce aux pratiques culturelles qui leurs sont associées, ce qui permet de les désigner comme des modèles de conservation de la nature (JuhéBeaulaton, 2002, 2005, 2006, 2010 ; Garcia et al., 2006 ; Hamberger, 2010 ; Kokou et Kokuste, 2010 ; Liberski- Bougnoud et al., 2010 ; Kaboré, 2010 ; Boukpessi, 2003, 2010). 
Malgré leur petite superficie, ces bois sacrés dans leur majorité se sont révélés comme un outil important de conservation de la biodiversité. En effet, ils constituent une sorte de pépinières sur site et des réservoirs génétiques. Ils renferment également de nombreuses plantes médicinales, des espèces animales et végétales en danger, de gros arbres considérés comme sacrés, de plusieurs arbres fruitiers qui attirent l'avifaune et autres animaux sauvages (Kokou et al., 2005 ; Adou Yao el al., 2011). La sacralisation de ces sites constituerait une nouvelle stratégie efficace à inclure dans les processus de gestion moderne de la biodiversité (Soury, 2007). Malheureusement, le pouvoir des chefs traditionnels et des dignitaires et l'occultisme des divinités installées dans ces forêts ont considérablement baissé de sorte que les forêts sacrées sont, depuis quelques années, soumises à l'exploitation anarchique et à d'autres facteurs de destruction (Adjossou, 2004). Aujourd'hui, l'expansion des religions révélées et le pouvoir des gestionnaires de ces lieux sacrés se dégradent. Dans la Commune de So-Ava, les bois sacrés qui étaient entre temps préservés, actuellement $0,4 \%$ de bois de feu y sont prélevés (Ajdjakpa et al., 2009). Face à l'explosion démographique, les stratégies traditionnelles de conservation de la biodiversité sont mis à mal par l'occupation horizontale des terres (Kokou et al., 2005 ; Kokou et Kokutse, 2006 ).

$\mathrm{Au}$ Centre Bénin, le diagnostic sur la conservation de la biodiversité par les bois sacrés dresse un tableau relativement pessimiste. Ce travail vise à analyser l'influence des pressions anthropiques sur la biodiversité des bois sacrés du Centre Bénin.

\section{MATERIEL ET METHODES}

\section{Milieu d'étude}

Le Centre Bénin est situé entre $2^{\circ}$ et $2^{\circ}$ 46 de longitude Est et entre $7^{\circ} 30$ et $9^{\circ}$ de latitude Nord. Il couvre entièrement les
Communes de Glazoué, de Savè et de Ouèssè (Figure 1)

\section{Etudes phytosociologiques}

Les relevés phytosociologiques sont effectués suivant la méthode de BraunBlanquet (1932). Cette méthode est basée sur le principe d'homogénéité floristique de la surface étudiée. Les placettes installées ont une dimension de $30 \mathrm{~m} \times 30 \mathrm{~m}\left(900 \mathrm{~m}^{2}\right)$. Au total, 88 placettes ont été installées.

\section{Estimation de la pression pastorale}

Elle est estimée de façon indirecte en utilisant :

- premièrement, la densité de fèces des ruminants (DF), aussi bien de l'année que des années antérieures par des relevés. Cette densité est divisée en trois classes (Classe 1 : $\mathrm{DF} \leq 1 \%$, Classe $2: 1<\mathrm{DF} \leq 20 \%$, Classe 3 : DF $>20 \%$ ) ;

- deuxièmement, trois classes sont également considérées pour estimer la densité du piétinement (DP) (Classe $1: \mathrm{DP} \leq 1 \%$, Classe 2: $1<\mathrm{DP} \leq 50 \%$, Classe 3 : DP > 50\%) ;

- troisièmement, les dégâts des animaux sur les plantes fourragères (DV) sont codés en trois classes (Classe 1) pour les stations sur lesquelles la flore fourragère (herbacée comme ligneuse) présente très peu de traces de prélèvements, Classe 2 pour celles sur lesquelles ces traces ont porté sur moins de la moitié des plantes présentes, Classe 3 pour les stations sur lesquelles plus de $50 \%$ des organes de végétaux fourragers ont présenté des traces de prélèvement. L'indice de la pression pastorale apparente (IP) est obtenu par la somme de ces trois indices (IP $=\mathrm{DF}+$ $\mathrm{DP}+\mathrm{DV})$. Les placettes sont classées en trois catégories : placettes non ou faiblement pâturées (IP $\leq 3$ ), placettes moyennement pâturées $(3<$ IP $\leq 6$ et placettes surpâturées (IP > 6). Cet indice est utilisé en 2002 au nord du Cameroun (Onana et Devineau, 2002). 


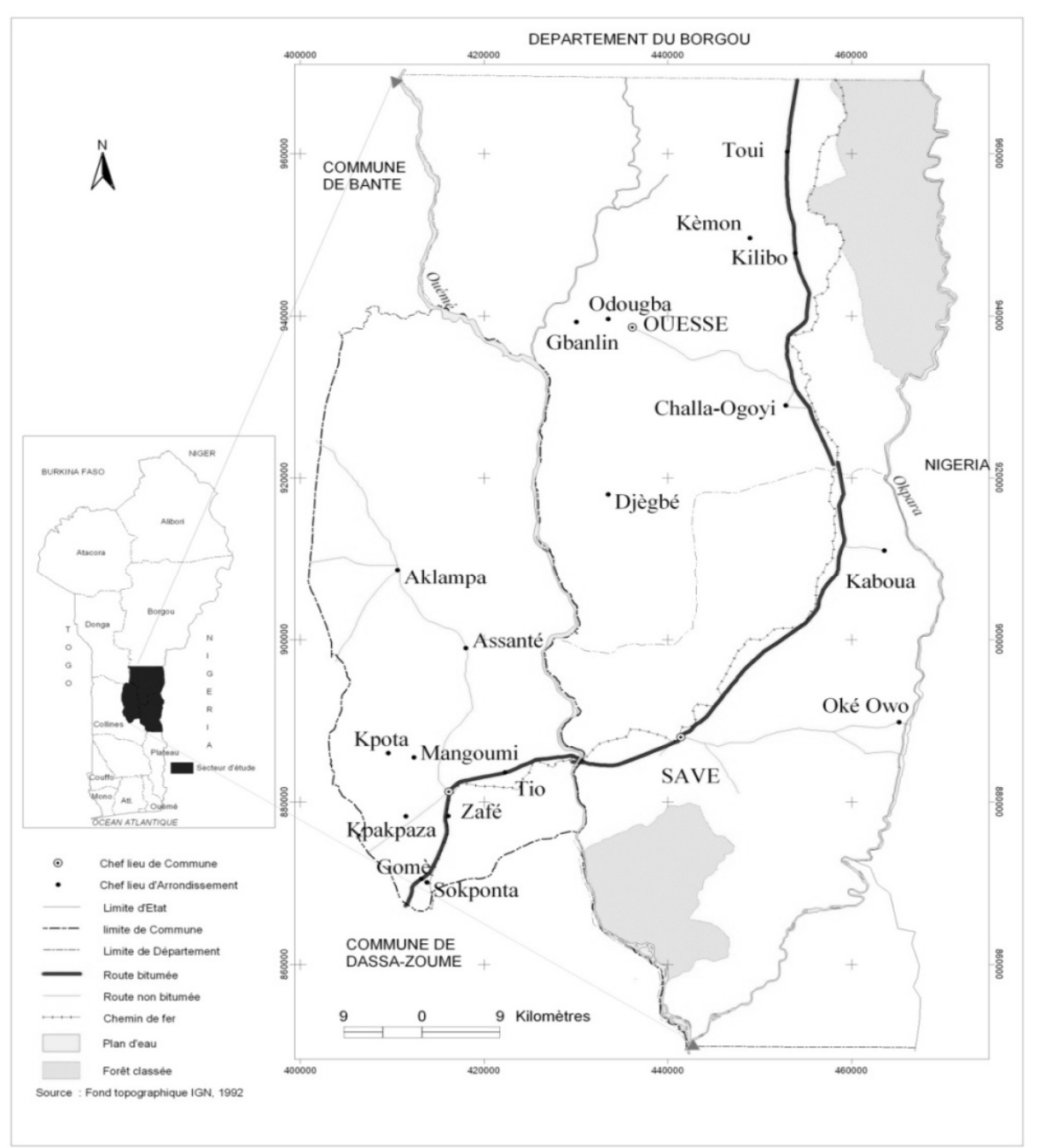

Figure 1: Situation géographique du Centre.

\section{Enquêtes socio-économiques}

Quatre catégories de personnes ont été choisies pour les enquêtes : des agriculteurs, des dignitaires, des gestionnaires des bois sacrés et des Praticiens de la Médecine Traditionnelle. Les enquêtés sont pour la plus part animistes et chrétiens, à 97\% autochtones. Ils appartiennent au groupe socioculturel, Idatcha, Tchabè, Mahi et Fon. Les enquêtés sont choisis de façon raisonnée dans le village qui habite le bois sacré, une personne par catégorie et 61 bois sacrés. Au total 244 personnes riveraines ont été enquêtées, âgées d'au moins 30 ans.

\section{RESULTATS \\ Rituel dans les bois sacrés}

Les enquêtés ont fait savoir que les bois sacrés sont des temples d'initiation, de méditation, de libation pour recevoir divers messages et offres divins. Tous les adeptes n'ont pas accès à certains sites dans les bois sacrés. L'accès à certains lieux sacrés est fonction du degré d'initiation (niveau spirituel). L'accès aux bois sacrés de la divinité Oro (Igbo Oro) se fait à reculons par une entrée faite de rameaux tissés, porte d'accès servant de filtre spirituel des énergies. 


\section{Utilisation locale de certaines ressources floristiques et fauniques}

Certaines espèces des bois sacrés sont exploitées à des fins socio-économiques. Elles le sont pour des besoins médicaux, alimentaires, bois d'énergie, bois de services, etc. La médecine traditionnelle utilise certaines parties des ligneux tels que: les racines, l'écorce (Photo 1), les feuilles et les fruits pour le traitement de certaines maladies.

L'utilisation de la faune est réalisée à travers le prélèvement de certains organes comme : la tête, la peau, la corne, les dents, etc.. Ces organes prélevés permettent de guérir en association avec d'autres plantes ou non certaines maladies et aussi à des fins médico-magiques. Les Tableaux 1 et 2 présentent le rôle ethnozoologique et ethnobotanique de quelques espèces. L'utilisation des espèces floristiques varie par communauté. L'intérêt accordé à ces ressources est lié à leur disponibilité. Le Tableau 2 présente les différents usages de quelques espèces floristiques. Les bois sacrés sont perçus comme une partie importante du patrimoine des villages. Certains sont importants à tel point que leur disparition peut entraîner des perturbations sociales.

C'est le cas du bois sacré Idotché en plein cœur du village de Toui (Photo 2) qui en disparaissant ne reste que l'arbre Adansonia digitata. De façon mystérieuse,

Idotché protège tous les habitants de Toui. Il permet aux couples d'avoir des enfants. Certaines plantes médicinales des bois sacrés constituent une immense réserve, surtout celles qui sont rares. Ainsi, non seulement ces bois sacrés contribuent à la santé publique mais aussi à l'économie des ménages.

\section{Mode de gestion des bois sacrés}

Selon la plupart des dignitaires, les bois sacrés sont des lieux de transfert de savoirs endogènes aux générations futurs. Les bois qui abritent la divinité Oro sont confiés au chef des dignitaires «Adjina » désigné par le comité des sages. Il sert de trait d'union entre les divinités et les autres adeptes. La désignation du gestionnaire se fait de deux manières : soit par la consultation de l'oracle Fâ ou consensuelle.

Des $75 \%$ des bois sacrés étudiés, le gestionnaire est désigné parmi les adeptes ou par la consultation de l'oracle Fâ. Dans la Commune de Ouèssè, le bois sacré Winman est géré par la cour royale.

Quelques bois sacrés actuels doivent leur existence à la législation traditionnelle qui est crainte. Aujourd'hui, les personnes qui jouent ce rôle appartiennent à une classe d'âge qui disparaît. Les jeunes non initiés accordent peu d'intérêt aux bois sacrés. Le décès d'un prêtre d'une divinité de bois sacrés peut conduire à son défrichement. Certains riverains évoquent le régime révolutionnaire Marxiste-Léniniste des années 80 au Bénin, responsable de l'abattage des grands arbres de ces lieux, sous le prétexte qu'ils servent de lieu de rencontre et de refuge des sorciers. Cet état de fait décrété par le gouvernement d'alors a favorisé la réduction des superficies des bois sacrés et la disparition d'autres. Aujourd'hui, la prolifération des églises ainsi que les messages destructeurs des valeurs ancestrales véhiculés au sein des populations provoquent un désintéressement des jeunes aux valeurs ancestrales.

\section{Formes de pression sur la biodiversité des bois sacrés}

Actuellement, c'est dans les bois sacrés qu'on retrouve encore certaines espèces très utiles, assez rares et menacées. A l'intérieur de ces bois, on note par endroits des arbres écorcés, des racines prélevées de même que des feuilles des espèces pour le traitement de certaines maladies. Les Praticiens de la Médecine Traditionnelle (PMT) utilisent des ossements et/ou des cadavres de quelques animaux et oiseaux dans le traitement des maladies ou à des fins médico-magiques. La plupart des enquêtés ont cité l'homme et ses activités comme principale cause de destruction des bois sacrés. Ainsi, ces écosystèmes perdent leur superficie originale 
et sont complètement réduits à quelques grands arbres. Un tel habitat ne peut plus servir de refuge à la faune. Les populations locales reconnaissent que certaines espèces qui vivaient dans les bois sacrés n'y sont plus ou ont migré vers d'autres habitats plus propices à leur survie. La chasse et la coupe persistent encore dans certains bois sacrés (Photo 3).

Espèces menacées de disparition dans les bois sacrés

Compte tenu des différentes pressions sur les bois sacrés, certaines espèces sont menacées de disparition. Plus de $90 \%$ des enquêtés ont cité Milicia excelsa comme espèce dangereusement menacée de disparition. Des espèces comme Afzelia africana, Antiaris toxicaria, Dracaena arborea, Adansonia digitata et Pterecarpus erinaceus sont aussi menacées de disparition par la pression anthropique. Les familles les plus menacées se présentent dans le Tableau 3. Dans les bois sacrés, les familles qui subissent plus de pression sont les Bignoniaceae, Fabaceae et Mimosaceae. La pression est faible sur les autres familles.
Toute la faune sauvage des bois sacrés est considérée comme menacée de disparition. On peut citer en priorité Trionomys swinderianus, Varanus exanthematicus, Varanus niliticus, Python sebae, Dendrohyrax dorsalis, Manis tricuspide, Ourebia ourebi, Cercopithecus chlorocebus, Aethiops tantalus, Colobus vellerosus, Papio anubis, Pelusios casteneus, Civettictis civetta, Hystrix cristata, Syncerus caffer brachyceros, Xerus erythropus et Trichechus senegalensis que l'on ne retrouve plus dans certains villages.

\section{Indice de pression pastorale dans les bois sacrés}

Du Tableau 4, il ressort que l'indice de pression pastorale est faible dans tous les bois sacrés. Ils sont sous pression pastorale : IP $\leq 3$ dans 14 bois sacrés ; IP moyenne dans 9 bois sacrés ( $3<$ IP $\leq 6=9$ ) ; IP forte dans 6 bois sacrés (IP > $6=6$ ). Au Bénin, les placettes installées dans 61 bois sacrés ont montré que 29 sont sous la pression des bovins. Le Tableau 5 permet d'apprécier le statut de certaines espèces par rapport à la liste rouge de l'UICN.

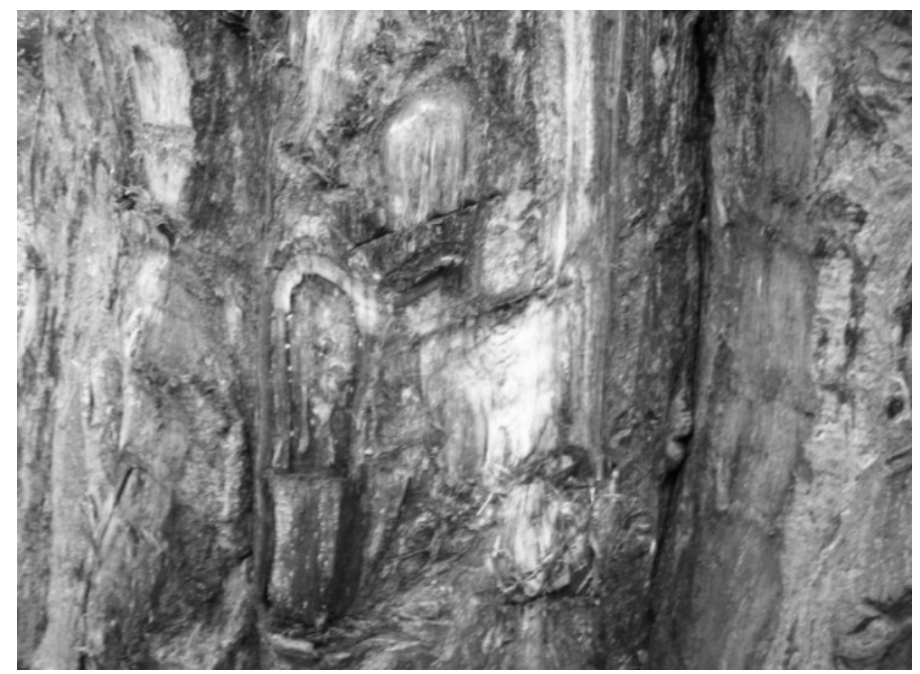

Photo 1: Ecorçage de 1'arbre Adansonia digitata L. dans le Bois sacré Covèzoun. 


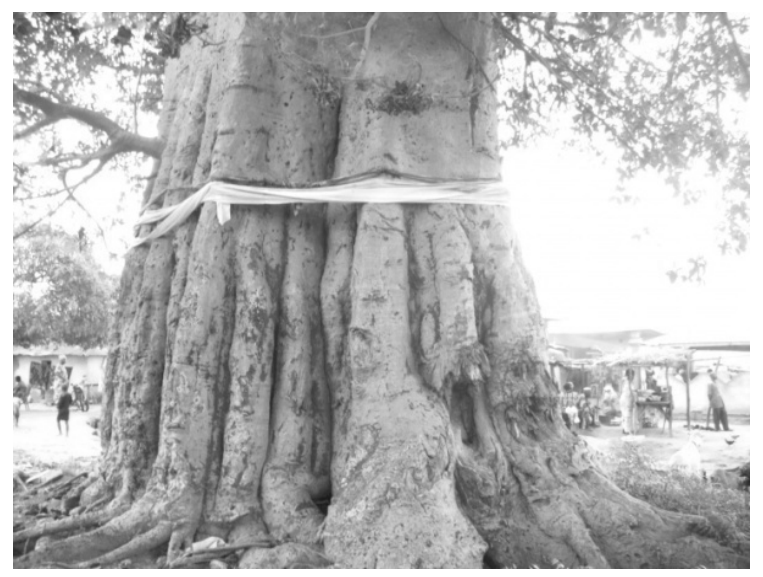

Photo 2: Adansonia digita L. sacré en plein cœur du village de Toui au Centre Bénin.

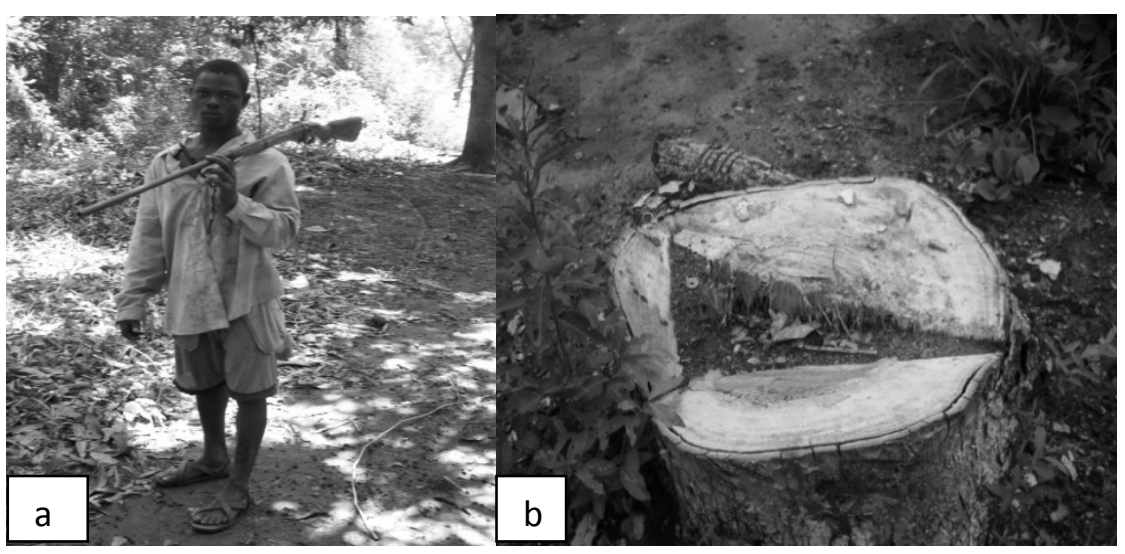

Photo 3: Chasseur surpris dans le bois sacré Igbo Aira Aira (a) ; coupe de Daniella oliveri dans le bois sacré Covèzoun (b).

Tableau 1: Usages locaux de quelques espèces animales des Bois sacrés du Centre Bénin.

\begin{tabular}{|c|c|c|c|}
\hline Espèces & Organes utilisés & Usages & Pression \\
\hline Bitis arietans Merrem & Tête + dents & $\begin{array}{l}\text { Médico-magique; morsure de } \\
\text { serpent }\end{array}$ & ++ \\
\hline Cephalophus niger Gray & $\begin{array}{l}\text { Tête ; urine ; Os de } \\
\text { patte + ingrédients }\end{array}$ & $\begin{array}{l}\text { Vertige ; maux d'oreille ; } \\
\text { Accélère la marche du bébé }\end{array}$ & +++ \\
\hline $\begin{array}{l}\text { Cercopithecus mona } \\
\text { Waterhouse }\end{array}$ & Tête & Facilite l'accouchement & +++ \\
\hline Civettictis civetta Schreber & Glande de la fesse ; tête & $\begin{array}{l}\text { Facilite la dentition du } \\
\text { bébé ; médico- magique }\end{array}$ & +++ \\
\hline Cricetomys gambianus & $\begin{array}{l}\text { Intestin (séché et } \\
\text { écrasé) }\end{array}$ & Médico-magique & ++ \\
\hline Crocodilus niloticus & Dents + ingrédients & Facilite la dentition du bébé & +++ \\
\hline $\begin{array}{l}\text { Eidolon helvum ; Pteropus } \\
\text { comorensis }\end{array}$ & Entière & Facilite l'accouchement & +++ \\
\hline
\end{tabular}


R. I. EHINNOU KOUTCHIKA et al. / Int. J. Biol. Chem. Sci. 7(1): 306-318, 2013

\begin{tabular}{lllc}
\hline Erythrocebus patas Schreber & Tête + peau & Vertige & +++ \\
\hline Manis tricuspide & Ecaille + ingrédients & Convulsions & +++ \\
\hline Naja melanoleuca Hallowel & Tête & Miner la femme $;$ fertilité & ++++ \\
\hline Python sebae Gmelin & Dents & Facilite la dentition du bébé & ++++ \\
\hline Tragelaphus scriptus Pallas & Graisse & Guérison de maladies & ++ \\
\hline \multicolumn{2}{c}{ Pression anthropique: $++++=$ très forte $;+++$ forte $;++=$ moyenne $;+=$ faible } &
\end{tabular}

Tableau 2: Usages locaux de quelques espèces floristiques des bois sacrés du Centre Bénin.

\begin{tabular}{|c|c|c|c|c|}
\hline Espèces & Familles & $\begin{array}{c}\text { Organes } \\
\text { utilisés }\end{array}$ & Usages & Pression \\
\hline $\begin{array}{l}\text { Afzelia africana } \text { Smith ex } \\
\text { Pers. }\end{array}$ & Caesalpiniaceae & Ecorce & $\begin{array}{l}\text { Maux de ventre, bois } \\
\text { d'œuvre et bois énergie }\end{array}$ & ++++ \\
\hline $\begin{array}{l}\text { Anchomanes difformis } \\
\text { (Blume) Engl. }\end{array}$ & Araceae & Racine & Erythèmes fessiers & + \\
\hline Antiaris toxicaria Lesch. & Bignoniaceae & Ecorce & $\begin{array}{l}\text { Maux de ventre, } \\
\text { panaris, stérilité } \\
\text { féminine, bois d'œuvre } \\
\text { et bois énergie }\end{array}$ & ++ \\
\hline Antidesma venosum Tul. & Annonaceae & Racine & $\begin{array}{l}\text { Maux de dent et bois } \\
\text { énergie }\end{array}$ & + \\
\hline Azadirachai ndica A. Juss. & Asteraceae & Jeune feuille & $\begin{array}{l}\text { Paludisme, hernie, } \\
\text { maux de ventre et bois } \\
\text { énergie }\end{array}$ & ++++ \\
\hline Blighia sapida Koenig & Bignoniaceae & Feuille, fruit & $\begin{array}{l}\text { Paludisme, alimentaire } \\
\text { et savon }\end{array}$ & + \\
\hline Bridelia ferriginea Benth. & Boraginaceae & Feuille & $\begin{array}{l}\text { Maux de ventre et bois } \\
\text { énergie }\end{array}$ & ++++ \\
\hline Ceiba pentandra (L.)Gaertn. & Bombacaceae & Epine/Ecorce & $\begin{array}{l}\text { Dentition du bébé, bois } \\
\text { d'œuvre et bois énergie }\end{array}$ & ++ \\
\hline Cola gigantea A.Chev. & Sterculiaceae & Feuille sèche & $\begin{array}{l}\text { Règle douloureuse, } \\
\text { tremblote, bois d'œuvre } \\
\text { et énergie }\end{array}$ & ++ \\
\hline Cordia senegalenses Juss. & Convolvulaceae & Feuille & Anémie & + \\
\hline Dialium guineense Willd. & Convolvulaceae & $\begin{array}{l}\text { Feuille, } \\
\text { écorce, racine }\end{array}$ & $\begin{array}{l}\text { Maux de ventre, fièvre, } \\
\text { paludisme, hernie, } \\
\text { ulcère ; bois énergie et } \\
\text { bois d'œuvre }\end{array}$ & ++ \\
\hline $\begin{array}{l}\text { Dicrostachys cinerea } \\
\text { (L.)Wight \& Arn. }\end{array}$ & Mimosaceae & Tige & $\begin{array}{l}\text { Brosse végétale, lutte } \\
\text { contre la carie dentaire }\end{array}$ & ++ \\
\hline $\begin{array}{l}\text { Dracena arborea } \\
\text { (Willd.)Link }\end{array}$ & Bignoniaceae & Ecorce & $\begin{array}{l}\text { Diarrhée, anémie, } \\
\text { angine, maux de ventre, } \\
\text { maux de tête et bois } \\
\text { d'œuvre }\end{array}$ & + \\
\hline Elaeis guineensis Jacq. & Ebenaceae & Racine & $\begin{array}{l}\text { Vertige, alimentaire, } \\
\text { bois d'œuvre et bois } \\
\text { énergie }\end{array}$ & +++ \\
\hline Ficus exasperata Vahl & Fabaceae & Feuille & $\begin{array}{l}\text { Toux, diarrhée et bois } \\
\text { énergie }\end{array}$ & +++ \\
\hline Ficus тисиso Ficalho & Fabaceae & Feuille & Anémie et bois énergie & ++ \\
\hline Ficus sur Forssk. & Fabaceae & Ecorce & Fièvre, bois énergie & +++ \\
\hline
\end{tabular}


R. I. EHINNOU KOUTCHIKA et al. / Int. J. Biol. Chem. Sci. 7(1): 306-318, 2013

\begin{tabular}{|c|c|c|c|c|}
\hline Ficus vogelina (Miq.)Miq. & Fabaceae & Feuille & $\begin{array}{l}\text { Hémorroïde et bois } \\
\text { énergie }\end{array}$ & ++ \\
\hline Momordica charantia L. & Cucurbitaceae & Feuille & Varicelle et bois énergie & ++ \\
\hline Morinda lucida Benth. & Rubiaceae & Ecorce & $\begin{array}{l}\text { Faiblesse sexuelle, } \\
\text { paludisme et bois } \\
\text { d'œuvre }\end{array}$ & + \\
\hline $\begin{array}{l}\text { Newbouldia laevis (P.Beauv.) } \\
\text { Seem. }\end{array}$ & Bignoniaceae & $\begin{array}{l}\text { Ecorce, } \\
\text { feuille }\end{array}$ & $\begin{array}{l}\text { Maux de ventre, } \\
\text { vertige ; paludisme, } \\
\text { purification et bois } \\
\text { énergie }\end{array}$ & + \\
\hline $\begin{array}{l}\text { Parkia bigloboza (Jacq.)R.Br. } \\
\text { ex G.Don f. }\end{array}$ & Mimosaceae & Ecorce et fruit & Toux et alimentaire & ++ \\
\hline Spondia monbin L. & Anacardiaceae & Ecorce & $\begin{array}{l}\text { Paludisme, alimentaire } \\
\text { et bois énergie }\end{array}$ & ++ \\
\hline $\begin{array}{l}\text { Vernonia colorata (Willd.) } \\
\text { Drake }\end{array}$ & Verbenaceae & Ecorce+racine & $\begin{array}{l}\text { Hémorroïde, alimentaire } \\
\text { et bois énergie }\end{array}$ & + \\
\hline Vtitex doniana Sweet & Vitaceae & Ecorce & $\begin{array}{l}\text { Hémorroïde, } \\
\text { alimentaire et bois } \\
\text { énergie }\end{array}$ & +++ \\
\hline
\end{tabular}

Pression anthropique: $\quad++++=$ très forte $; \quad+++=$ forte $; \quad++=$ moyenne $; \quad+=$ faible

Tableau 3: Pression anthropique de certaines familles des espèces floristiques menacées dans les bois sacrés du Centre Bénin.

\begin{tabular}{lcc}
\hline Familles & Fréquence & Pression \\
\hline Anacardiaceae & 1 & + \\
Annonaceae & 1 & + \\
Araceae & 1 & + \\
Astéraceae & 1 & + \\
Bignoniaceae & 4 & ++++ \\
Bombacaceae & 1 & + \\
Boraginaceae & 1 & + \\
Caesalpiniaceae & 1 & + \\
Convolvulaceae & 1 & + \\
Cucurbitaceae & 1 & + \\
Ebénaceae & 1 & + \\
Fabaceae & 4 & ++++ \\
Mimosaceae & 2 & ++ \\
Rubiaceae & 1 & + \\
Sterculiaceae & 1 & + \\
Verbénaceae & 1 & + \\
Vitaceae & 1 & + \\
\hline \multicolumn{1}{c}{ Pression anthropique: $++++=$ très forte } & $+++=$ forte $;++=$ moyenne $;+$ faible.
\end{tabular}


Tableau 4: Estimation de la pression pastorale apparente sur les bois sacrés à partir des densités de fèces, du piétinement et des dégâts sur la flore fourragère.

\begin{tabular}{|c|c|c|c|c|}
\hline \multirow{2}{*}{ Bois sacrés } & \multicolumn{3}{|c|}{ Facteurs de classes } & \multirow{2}{*}{ IP } \\
\hline & DF & DP & $\overline{\text { DV }}$ & \\
\hline Aboklémè & 2 & 2 & 0 & 4 \\
\hline Aimoudji & 3 & 3 & 3 & 9 \\
\hline Atchin & 1 & 2 & 3 & 6 \\
\hline Bossikponzoun & 3 & 2 & 1 & 6 \\
\hline Covèzoun & 3 & 3 & 2 & 8 \\
\hline Dankamè & 3 & 3 & 3 & 9 \\
\hline Djèzoun & 1 & 1 & 0 & 2 \\
\hline Ekiti & 2 & 2 & 0 & 4 \\
\hline Ghézoun & 2 & 2 & 2 & 6 \\
\hline Ibaba Guidai & 2 & 2 & 0 & 4 \\
\hline Igbo aira & 1 & 0 & 0 & 1 \\
\hline Igbo Etou & 1 & 1 & 0 & 2 \\
\hline Igbo kori & 1 & 0 & 0 & 1 \\
\hline Igbo Oro de Alafia & 2 & 1 & 1 & 4 \\
\hline Igbo Oro de Attèssè & 2 & 1 & 0 & 3 \\
\hline Igbo Oro de Odélakou & 1 & 1 & 0 & 2 \\
\hline Igbo Oro de Ogoutèdo & 2 & 1 & 0 & 3 \\
\hline Iya & 0 & 1 & 0 & 1 \\
\hline Kokoyazoun & 3 & 3 & 2 & 8 \\
\hline Lagbézoun & 3 & 3 & 3 & 9 \\
\hline Monozoun & 3 & 2 & 3 & 8 \\
\hline Odjoulè & 1 & 1 & 1 & 3 \\
\hline Orozou de Botti & 1 & 0 & 0 & 1 \\
\hline Orozoun de Aklamkpa & 1 & 1 & 1 & 3 \\
\hline Orozoun de Assanté & 1 & 0 & 1 & 2 \\
\hline Orozoun de Banigbé & 1 & 0 & 2 & 3 \\
\hline Tchèzounmè & 1 & 0 & 0 & 1 \\
\hline Vèzoun & 2 & 2 & 2 & 6 \\
\hline Wlazounzan & 1 & 3 & 3 & 6 \\
\hline
\end{tabular}


Tableau 5: Appréciation de certaines espèces par rapport à la liste rouge de l'UICN et leur statut au Bénin.

\begin{tabular}{lccc}
\hline Espèces & Famille & $\begin{array}{c}\text { Liste rouge } \\
\text { UICN }\end{array}$ & $\begin{array}{c}\text { Statut } \\
\text { au Bénin }\end{array}$ \\
\hline Afzelia africana Smith ex Pers. & Caesalpinoideae & VU & EN \\
Borassus aethiopum Mart. & Arecaceae & - & VU \\
Khaya senegalensis (Ders.) A. Juss. & Meliaceae & VU & EN \\
Milicia excelsa (Welw.) C.C.Berg & Moraceae & VU & EN \\
Ptereocarpus erinaceus Poir. & Papilionoideae & - & EN \\
Vitellaria paradoxa Gaertn F. & Sapotaceae & VU & VU \\
Zanthoxylum zanthozyloides & Rutaceae & - & VU \\
(Lam.)Zepernick \& Timber & & & \\
\hline Catégorie UICN : VU= vulnérable; Statut au Bénin : EN = en danger, VU= vulnérable. &
\end{tabular}

\section{DISCUSSION}

L'analyse des marques d'activités humaines montre que certains bois sacrés sont soumis à une très forte pression de la part des riverains. Contrairement à ce qui est affirmé dans la littérature (Hamberger, 2010 ; Kokou et Kokuster, 2010), les bois sacrés ne sont pas des sanctuaires inviolés. Les interdits et les croyances n'empêchent pas les habitants de rentrer et de prélever du bois de feu, du bois d'œuvre, des produits forestiers non ligneux, etc. (Adjakpa et al., 2009). Ces perturbations fréquentes modifient la structure forestière, qui diffère de plus en plus de la canopée dense et fermée d'une forêt naturelle (Garcia et al., 2006 ; Abdou, 2006).

Malgré ce rôle de protection d'espèces menacées ou vulnérables, la pression que ces bois sacrés subissent est très forte. On note que certaines espèces ménacées de disparition comme, Afzelia africana, Borassus aeithiopum, Khaya senegalensis, Milicia excelsa, Ptereocarpus, Vitellaria paradoxa et Zanthoxylum zanthozyloides signalées par Adomou (2005), se retrouvent dans les bois sacrés. Ces reliques permettent de conserver la biodiversité (Garcia, 2003; Bhagwat, 2005 ; Garcia et al., 2006 ; Soury,
2007). Dans la plupart des cas, leur structure verticale est complètement perturbée. Les grands arbres sont abattus provoquant la descente de cime entraînant l'embroussaillement du sous-bois, qui devient impénétrable. La proportion de lianes dans ces forêts est identique à celle trouvée dans les îlots forestiers du Togo (Kokou et al., 2002 ; Kokou et Caballé, 2005). En Inde, Swamy et al. (2003) ont montré que l'invasion d'espèces étrangères est devenue un problème grave pour l'écologie de certains bois sacrés car elles menacent et déciment souvent les espèces locales.

Cette étude illustre aussi la fragilité actuelle des systèmes de gestion locale des bois sacrés que les populations avaient ellesmêmes protégées à travers plusieurs générations. Des conflits peuvent éclater et conduire à la dégradation ou à la disparition d'un bois sacré. Les éleveurs traversent ces bois quotidiennement avec leur troupeau pour les besoins de pâturage. Les conséquences de la destruction complète de ces bois sacrés se traduiraient par une perte de la biodiversité et l'extinction de quelques espèces clés. Dès lors, il paraît urgent de renforcer les capacités locales, le plus rapidement possible, pour éviter la 
disparition totale de ces reliques de bois et de leur diversité biologique. Certes la gestion locale de ces bois sacrés montre ses limites mais les populations manifestent toujours une volonté de les préserver. Sans cette volonté, tous ces bois auraient complètement disparus. Plusieurs auteurs ont évoqué la nécessité de tirer certaines leçons de ces systèmes de gestion locale (Djah, 2009). Mais les problèmes et conflits relevés dans cette étude (croissance démographique, expansion incontrôlée des habitations, érosion des croyances religieuses et faiblesse actuelle du pouvoir des chefs religieux) vont toujours se poser, menaçant ainsi l'avenir des bois sacrés.

\section{Conclusion}

Les bois sacrés permettent de mesurer la forme endogène de conservation de la biodiversité dans le Centre Bénin. Aujourd'hui, l'avenir de la plupart des bois sacrés sombre face à l'évolution démographique et l'ascension des religions révélées. Depuis quelques années, le pouvoir de certains dignitaires est mis à mal face à l'intensification des prélèvements pour des raisons médicinales, de bois d'œuvre et de bois énergie. Il est donc impérieux de trouver d'autres alternatives pour la sauvegarde de la biodiversité des bois sacrés face à l'assaut des riverains et des éleveurs transhumants. Les bois doivent être pris en compte dans des projets de développement par les autorités au plus haut niveau que par les élus locaux dans le contexte actuel de transfert de compétence à la base.

\section{REFERENCES}

Adjossou K. 2004. Diversité floristique des forêts riveraines de la zone écologique IV du Togo. Mém. DEA biologie de développement, option biologie végétale appliquée, Univ. Lomé, p.75.
Adomou A. 2005. Vegetation patterns and environmental gradients in Bénin. Implications for biogeography and conservation. PhD Thesis, Wageningen University, Wageningen, p. 136.

Abdou R. 2006. Evaluation de la régénération naturelle ( $\mathrm{r} n$ ) des ligneux dans les champs de la zone sud du Département d'aguie : cas du terroir villageois de saja manja. Mémoire de fin d'étude en vue de l'obtention du diplôme d'ingénieur des techniques agricoles, Université Abdou Moumouni du Niger, p. 70.

Adjakpa BJ, Ahoton EL,Wessie DMP, Akpo E, Chidikofan DMGF, Hounsinou ARSA. 2009. Exploitation, commercialisation et consommation du bois de feu dans les zones humides du Sud-Bénin : Cas de la Commune de SôAva. Climat et Développement, 8: 1425.

Adou Yao CY, Bakayoko A, Akpatou K B, N'Guessan K. 2011. Impacts de pressions anthropiques sur la flore et la structure de la végétation dans la forêt classée de Monogaga, Côte d'Ivoire. Journal of Animal \& Plant Sciences, 12(2): 1560-1572.

Bhagwat S, Kushalappa C, Williams P, Brown N. 2005. The role of informal protected areas in maintaining biodiversity in the Western Ghats of India. Ecology and Society, 10(1): 8.

Boukpessi T. 2003. Les pratiques endogènes de gestion et de conservation de la biodiversité : Cas des bois sacrés du Centre-Togo. Mémoire de géographie, Université de Lomé.

Boukpessi T. 2010. Les pratiques endogènes de conservation de la biodiversité au Centre-Togo. Thèse de doctorat unique en Géographie, Université de Lomé et de franche comité, p. 306. 
Braun-Blanquet J. 1932. Plant Sociology the Study of Plant Communities. Transfland revised and edited by Fullerg D, Conard HS; 439.

Djah M. 2009. Religion traditionnelle et gestion durable des ressources floristiques en Côte d'Ivoire : Le cas des Ehotilé, riverains du Parc National des Îles Ehotilé ». VertigO - la Revue Electronique en Sciences de l'Environnement, 9(2).

Essoh E. 2003. Plaider pour un patrimoine des bois sacrés en Afrique Noire. Actes $\mathrm{du} \mathrm{XII}^{\mathrm{e}}$ Congrès Forestier Mondial Québec Canada «La forêt, source de vie» Tome A «des forêts pour les gens ». FAO, p. 381.

Ganglo CJ. 2005. Groupements de sous-bois, identification et caractérisation des stations forestières : cas d'un bois au Bénin. Bois et Forêts des Tropiques, 285(3): 35-46.

Garcia CJ, Pascal P, Kushalappa GG. 2006. Les forêts sacrées du Kadagu en Inde: écologie et religion. Bois et Forêts des Tropiques, 288(2): 5-13.

Garcia C. 2003. Les forêts sacrées de Kodagu: valeur écologique, rôle social et implications pour la conservation de la biodiversité. Thèse de doctorat, université Claude-Bernard, Lyon 1, p.193.

Hamberger K. 2010. Perspectives de la brousse, la fonction symbolique des forêts sacrées en pays Ouachi (Sud-est Togo). In Forêts Sacrées et Sanctuaires Boisés des Créations Culturelles et Biologiques. Edition Karthala : BurkinaFaso;Togo; Bénin; 91-122.

Juhé-Baulaton D, Roussel B. 2002. Les sites religieux vodun: des patrimoines en permanente évolution. In Patrimonialiser la Nature Tropicale. Dynamiques Locales, Enjeux Internationaux, Collection Colloques et
Séminaires, Cormier-Salem M-C, JuhéBeaulaton D, Boutrais J, Roussel B (eds). IRD: Paris; 415-438.

Jué-Beaulaton D. 2005. Enjeux économiques et sociaux autour des bois sacrés et la « conservation de la biodiversité » Bénin, Burkina Faso et Togo. Actes de l'atelier IFB, 2006: Dynamique de la biodiversité et modalité d'accès aux milieux et aux ressources, Fréjus, p.6879.

Jué-Beaulaton D. 2006. Enjeux économiques et sociaux autour des bois sacrés et la « conservation de la biodiversité » Bénin, Burkina Faso et Togo. Actes de l'atelier IFB.

Jué-Beaulaton D. 2010. Forêts sacrées et sanctuaires boisés. Des créations culturelles et botaniques. Burkina Faso, Togo , Bénin, p. 280.

Kaboré A. 2010. Les stratégies communautaires d'adaptation au changement climatique: Cas des bois sacrés dans l'aire socioculturelle Moaaga du Burkina Faso. Thèse de doctorat unique en géographie, Université d'Abomey-Calavi, Bénin, p. 216.

Kokou K, Akoègninou A, Akpagana K. 2000. Analyse morpho-structurale et diversité floristique des ilots de forêt protégées dans la plaine côtière du sud $\mathrm{du}$ Togo. Etudes Flor. veg. Burkina Faso, 5: 33-48.

Kokou K, Caballé G. 2000. Les îlots forestiers de la plaine côtière togolaise. Bois et Forêts des Tropiques, 263(1): 3951.

Kokou K, Adjossou K, Hamberger K. 2005. Les forêts sacrées de l'aire ouatchi au sud-est du Togo et les contraintes actuelles des modes de gestion locale des ressources forestières. Vertig $O, 6(3)$.

Kokou K, Sokpon N. 2006. Les Forêts sacrées du couloir du Dahomey. Bois et forêts des tropiques, 288(2): 15-23. 
Kokou K, Kokuste A. 2010. Des forêts sacrées, dans la région du littorale très anthropisée du sud Togo. In Forêts Sacrées et Sanctuaires Boisés des Créations Culturelles et Biologiques. Edition Karthala: Burkina-Faso, Togo, Bénin ; 91 -122.

Liberski- Bougnoud D, Fourie A, Nigna S. 2010. Les "bois sacrés" faits et illusions à propos des santuaires boisés des Kasena (Burkina Faso). In Forêts Sacrées et Sanctuaires Boisés des Créations Culturelles et Biologiques. Edition Karthala: Burkina-Faso, Togo, Bénin; 59 -90.

Neuenschwander P, Sinsin B, Goergen G. 2011. Protection de la Nature en Afrique de l'Ouest: Une Liste Rouge pour le
Bénin. International Institute of Tropical Agriculture : Ibadan, Nigeria ; p. 365 .

Onana J, Devineau L. 2002. Afzelia africana Smith ex Persoon dans le NordCameroun. Etat actuel des peuplements et utilisation pastorale. Revue Élev. Méd. Vét. Pays Trop., 55(1): 39-45.

Soury A. 2007. Sacred forests: a sustainable conservation strategy? The case of sacred forests in the Ouémé Valley, Benin. MSc of International Development studies, Wageningen Univesity, p. 142.

Swamy PS, Kumar M, Sundarapandian SM. 2003. Spirituality and ecology of sacred groves in Tamil Nadu, India. Unasylva, 213(54): 53-58 